\title{
Modelagem Matemática e o Desenvolvimento do Pensamento
}

\section{Algébrico}

\author{
Thiago Fernando Mendes \\ Licenciado, Universidade Estadual do Norte do Paraná \\ thiagomendes_96@hotmail.com
}

Bárbara Nivalda Palharini Alvim Sousa Robim

Professora, Universidade Estadual do Norte do Paraná

barbara.palharini@uenp.edu.br

\section{Rudolph dos Santos Gomes Pereira}

Professor, Universidade Estadual do Norte do Paraná

rudolphsantos@uenp.edu.br

\section{Resumo}

Este artigo busca identificar características do pensamento algébrico, de alunos, que emergem no desenvolvimento de atividades de modelagem matemática. Fundamenta-se na modelagem matemática como alternativa pedagógica e na teoria do pensamento algébrico de Rômulo Campos Lins e Joaquim Giménez. A busca pelo entendimento da questão de pesquisa e a natureza dos dados coletados, por meio de registros escritos dos alunos, gravações em áudio e vídeo, bem como anotações em diário de campo, possibilitaram uma abordagem qualitativa e uma análise interpretativa com base na análise textual discursiva. A análise dos resultados possibilitou concluir que o desenvolvimento de uma atividade de modelagem matemática possibilita o desenvolvimento do pensamento algébrico, uma vez que nas etapas desta alternativa pedagógica as características do pensamento algébrico são evidenciadas.

Palavras-chave: Educação Matemática. Modelagem Matemática. Educação Algébrica. Pensamento Algébrico. Alternativa Pedagógica.

\section{Mathematical Modeling and Algebraic Thinking development}

\begin{abstract}
This paper aims to identify algebraic thinking characteristics' of students that emerge in the development of mathematical modeling activities. This study is based on mathematical modeling as an educational alternative, and the algebraic thinking theory proposed by Rômulo Campos Lins and Joaquim Giménez. The search for understanding the research question and the nature of data collected through written records of students, recording audio and video, and notes in a diary, allowed a qualitative approach and an interpretive analysis based on textual analysis discursive. Results allowed to conclude that the development of a mathematical modeling activity allows the development of algebraic thinking, since all of their characteristics were evidenced in the steps of this educational alternative.
\end{abstract}


Keywords: Mathematics Education. Mathematical Modeling. Algebraic Thinking. Algebraic Education. Educational Alternative.

\section{Introdução}

Ensinar matemática de forma que os alunos consigam produzir significados para conceitos matemáticos é foco de pesquisas no âmbito da Educação Matemática (PONTE, 2003; LINS, 2004; SKOVSMOSE, 2008).

Nesse contexto, a modelagem matemática visa contribuir com os processos de ensino e de aprendizagem de matemática e é apontada, por documentos oficiais, como alternativas para o professor em sala de aula (PARANÁ, 2008).

A educação em matemática compreende a educação algébrica, desde a Educação Básica ao Ensino Superior. Segundo Lins e Giménez (1997) dois são os objetivos centrais para a educação algébrica: permitir que os alunos sejam capazes de produzir significados para a álgebra e permitir que eles desenvolvam a capacidade de pensar algebricamente. Neste sentido, o desenvolvimento das habilidades técnicas deve ser uma consequência destes dois pontos centrais, e não precedê-los.

No Ensino Fundamental, ocorre a transição da aritmética para a álgebra. Para os autores supracitados, o grande objetivo da educação aritmética e algébrica deve ser encontrar o equilíbrio em três frentes: desenvolvimento da capacidade de pôr em jogo as habilidades de resolver problemas e até mesmo investigar e explorar situações; desenvolvimento de modos distintos de produzir significado; aperfeiçoamento das habilidades técnicas, ou seja, da capacidade de usar as ferramentas desenvolvidas com maior facilidade.

O entendimento de álgebra proposto na comunidade de pesquisadores, professores e educadores está para além de uma técnica composta por símbolos e letras, e vai ao encontro de uma atividade de generalização de relações, padrões e regras matemáticas, ou seja, "uma forma de pensamento e raciocínio acerca de situações matemáticas” (KIERAN, 2007, p. 5).

Nesse contexto, faz-se importante o desenvolvimento do pensamento algébrico de estudantes, bem como o contato com diferentes alternativas pedagógicas. Neste artigo, buscar-se-á identificar características do pensamento algébrico no desenvolvimento de atividades de modelagem matemática. 


\section{Referencial Teórico}

A pesquisa está baseada nos referenciais sobre modelagem matemática na educação matemática e pensamento algébrico.

Há cerca de três décadas modelagem matemática é alvo de pesquisas na educação matemática brasileira (BIEMBEMGUT, 2009; BARBOSA, 2001; ARAUJO, 2009; ALMEIDA, SILVA, VERTUAN, 2012; KLÜBER, 2013).

No âmbito internacional, diferentes perspectivas para a modelagem matemática foram delineadas por Kaiser e Sriraman (2006): modelagem realística ou modelagem aplicada; modelagem contextual; modelagem educacional, contemplando modelagem didática e modelagem conceitual; modelagem sócio-crítica; modelagem teórica ou epistemológica. Para além dessas perspectivas, os autores destacam uma meta-perspectiva, que trata de modelagem matemática do ponto de vista cognitivo e contempla:

a) Análise dos processos cognitivos que ocorrem durante os processos de modelagem e compreensão destes processos cognitivos.

b) Promoção de processos de pensamentos matemáticos usando modelos como imagens mentais ou até mesmo retratos psicológicos ou enfatizando a modelagem como processos mentais tais como a abstração ou generalização. (KAISER; SRIRAMAN, p. 304)

Ferri (2006) e Bean (2003) utilizaram a perspectiva cognitiva de modelagem matemática para tratar de tópicos associados à aprendizagem matemática de alunos.

Uma distinção é necessária quando se faz menção à modelagem matemática na educação matemática, se o foco é aprender a fazer modelagem matemática ou ensinar matemática utilizando de atividades de modelagem matemática. Essa distinção é contemplada em Galbraith (2012) por meio da explicitação de modelagem matemática como conteúdo e como veículo, respectivamente.

Considerar atividades de modelagem matemática como uma alternativa pedagógica em que se faz uma abordagem, por meio da matemática, de um problema não essencialmente matemático coincide com a orientação dos documentos oficiais brasileiros e é o entendimento sinalizado por Almeida e Brito (2005).

De modo geral, a atividade de modelagem matemática compreende fases relacionadas aos procedimentos necessários para analisar, estruturar e solucionar uma situação-problema: “inteiração, matematização, resolução, interpretação de resultados e validação" (ALMEIDA; SILVA; VERTUAN, 2012, p. 15). A inteiração consiste em inteirar-se a respeito do que está sendo desenvolvido; já a matematização está associada à transição da linguagem natural para a linguagem matemática, por meio de formulação de hipóteses, seleção de variáveis e simplificações. 
$\mathrm{Na}$ resolução ocorre a construção de $\operatorname{modelos}^{1}$ matemáticos de modo a solucionar os questionamentos e permitir inferências e conclusões sobre a situação inicial. A interpretação e validação dos resultados permite que os alunos apliquem os modelos obtidos e avaliem o processo de construção. Essas etapas nem sempre acontecem de forma linear, o que caracteriza a dinamicidade da atividade (ALMEIDA; SILVA; VERTUAN, 2012).

Dentre as diferentes perspectivas de Kaiser e Sriraman (2006), utilizamos a perspectiva cognitiva para embasar nossas reflexões sobre o desenvolvimento do pensamento algébrico de alunos enquanto desempenham atividades de modelagem matemática, bem como o entendimento de modelagem matemática como uma alternativa pedagógica para o ensino de matemática (ALMEIDA; BRITO, 2005) em direção à perspectiva de modelagem matemática como veículo (GALBRAITH, 2012).

As especificidades relacionadas ao ensino e à aprendizagem de conceitos matemáticos relacionados à álgebra, bem como ao desenvolvimento do pensamento algébrico, da Educação Básica ao Ensino Superior, suscita o interesse de diferentes pesquisadores (LINS; GIMÉNEZ, 1997; KAPUT, 1999; CYRINO; OLIVEIRA, 2011).

O raciocínio algébrico é necessário em vários campos da Matemática, como na aritmética, geometria e análise. Lins e Giménez (1997) denominam tal raciocínio como "pensamento algébrico" e afirmam que este pode ser considerado dependendo da concepção de álgebra que se tem: álgebra como aritmética generalizada; álgebra como um estudo de processos para resolução de problemas; álgebra como uma expressão de variação de grandezas; álgebra como um estudo de estruturas matemáticas.

Segundo Lins e Giménez (1997), o pensamento algébrico é um dos diferentes modos de produzir significado para a álgebra, o qual possui três características fundamentais, sendo: aritmeticismo, internalismo e analiticidade. O aritmeticismo está pautado na produção de significados somente para os números e operações algébricas. Já o internalismo consiste em considerar números e operações exclusivamente mediante suas propriedades. E a analiticidade refere-se à realização de operações sobre "[...] números desconhecidos como se fossem conhecidos" (LINS; GIMÉNEZ, 1997, p. 151).

Pensar algebricamente é sempre pensar operando segundo essas características. E, para que ocorra o desenvolvimento do pensamento algébrico, é necessária a realização de atividades em que os estudantes possam investigar regularidades, sistematizar propriedades, resolver e discutir problemas algébricos, modelar situações e estabelecer padrões entre diferentes informações (LINS; GIMÉNEZ, 1997).

\footnotetext{
${ }^{1}$ Modelo será assumido aqui como um conjunto de símbolos e relações matemáticas que representam situações, fenômenos ou objetos reais a serem estudados (FERRUZZI et al., 2004).
} 
De maneira similar, Carraher, Schliemann e Brizuela (2006) discutem o pensamento algébrico de forma a considerar as quatro operações básicas como funções. Dessa maneira, a álgebra é empregada como aritmética generalizada de números e quantidades, uma vez que a generalização é a 'essência' do pensamento algébrico.

Outro autor que discute o pensamento algébrico é Kaput (1999), referindo-se a esse pensamento como o ato de generalizar e expressar essa generalidade por meio de linguagens cada vez mais formais, sendo que tal pensamento pode acontecer de cinco formas distintas: generalização e formalização de padrões e de restrições; manipulação de formalismos guiada sintaticamente; o estudo de estruturas abstratas de cálculos e de relações; o estudo de funções, de relações e de variações conjuntas; utilização de múltiplas linguagens de modelação matemática e no controle de fenômenos.

O desenvolvimento do pensamento algébrico é fator fundamental para o domínio da álgebra e para o desenvolvimento em matemática dos indivíduos. No entanto, a abstração exigida para o desenvolvimento deste pensamento é colocada como sendo uma das dificuldades dos alunos (BARBOSA; BORRALHO, 2009; FIORENTINI; MIORIM; MIGUEL, 1993; FIORENTINI; FERNANDES; CRISTÓVÃO, 2005).

\section{Aspectos Metodológicos}

A identificação, por meio dos registros produzidos por alunos, das características do pensamento algébrico que emergem no desenvolvimento de atividades de modelagem matemática nos permite caracterizar o estudo como de natureza qualitativa e cunho interpretativo. Para ter acesso não só ao que os alunos explicitam por meio de seus registros, mas também ao que está implícito nestes registros, nos amparamos nos procedimentos da análise textual discursiva para análise dos dados (MORAES; GALIAZZI, 2007; LUCCAS, 2011).

A análise textual discursiva não pretende testar hipóteses para comprová-las ao final da pesquisa, mas compreender, reconstruir conhecimentos existentes sobre os temas investigados, de acordo com as etapas: desmontagens dos textos ou unitarização; estabelecimento de relações ou categorização; captação do novo emergente; o processo de auto-organização (MORAES; GALIAZZI, 2007).

A desmontagem dos textos ou unitarização implica em examinar o material que compõe o corpus da análise detalhadamente, fragmentando-os para atingir unidades constituintes, enunciados sobre os objetos ou fenômenos estudados. Após a unitarização, é necessário categorizar, estabelecer relações entre as unidades de base, combinando, classificando, reunindo os elementos unitários na formação de conjuntos que associam elementos próximos. Nesse processo, é possível a emergência 
de uma nova compreensão do todo, que possibilita a formulação de inferências e a criação de um metatexto, uma compreensão do produto da nova combinação dos elementos construídos nas outras etapas (MORAES; GALIAZZI, 2007).

O processo de auto-organização focaliza o ciclo todo. Os resultados finais, criativos e originais, não podem ser previstos, mas é indispensável o esforço para que a emergência do novo seja concretizada (MORAES; GALIAZZI, 2007).

Os dados analisados são provenientes do desenvolvimento de uma atividade de modelagem matemática elaborada pelos autores e desenvolvida por 9 alunos do terceiro ano do Ensino Médio, dos quais 7 foram selecionados para integrar o corpus dessa pesquisa, pois somente esses eram maiores de idade. Inicialmente, foi feita a organização, operacionalização e sistematização das ideias, bem como a codificação e enumeração do material selecionado. Para preservar a identidade dos alunos os códigos A1, A2, A3, A4, A5, A6 e A7 foram adotados.

Para o desenvolvimento da atividade foi entregue o texto Plantar árvores neutraliza emissão de gás carbônico ${ }^{2}$ sobre a compensação de $\mathrm{CO} 2$, juntamente com uma tabela contendo o consumo de energia elétrica ( $\mathrm{kWh} / \mathrm{mês})$, o $\mathrm{CO}_{2}$ emitido (t/ano) e a quantidade de árvores a serem plantadas (ano), cujas hipóteses eram de que os dados apresentados são exatos e o consumo de energia anual é constante em todos os meses. A situação-problema solicitava que, considerando os dados do texto descritos na tabela e as hipóteses, os alunos determinassem a quantidade máxima de $\mathrm{CO}_{2}$ que poderia ser emitida por ano, sem que fosse necessário o plantio de árvores para a compensação.

\section{Análises}

A estrutura geral da análise textual discursiva é composta pela categoria características do pensamento algébrico na modelagem matemática.

Essa categoria tem como objetivo analisar as características do pensamento algébrico evidenciadas nas resoluções apresentadas pelos alunos no decorrer do desenvolvimento da atividade de modelagem matemática. Essa categoria é composta por quatro unidades de análise definidas $a$ priori de acordo com as fases da modelagem matemática defendidas por Almeida, Silva e Vertuan (2012): inteiração; matematização; resolução; e interpretação e validação dos resultados. Busca-se, por meio dessas unidades de análise a identificação de características do pensamento algébrico, de alunos, que possam emergir nas fases da modelagem matemática. Buscando contemplar o objetivo da pesquisa, cada unidade de análise foi subdividida em três subunidades relacionadas às características do pensamento algébrico contempladas na literatura (Figura 1).

\footnotetext{
${ }^{2}$ Fonte: SPILIMBERGO, J. Plantar árvores neutraliza emissão de gás carbônico. Disponível em: 〈www.gaz.com.br〉. Acesso em 17.08.2013 - 23:00h.
} 
Figura 1 - Características do Pensamento Algébrico na Modelagem Matemática

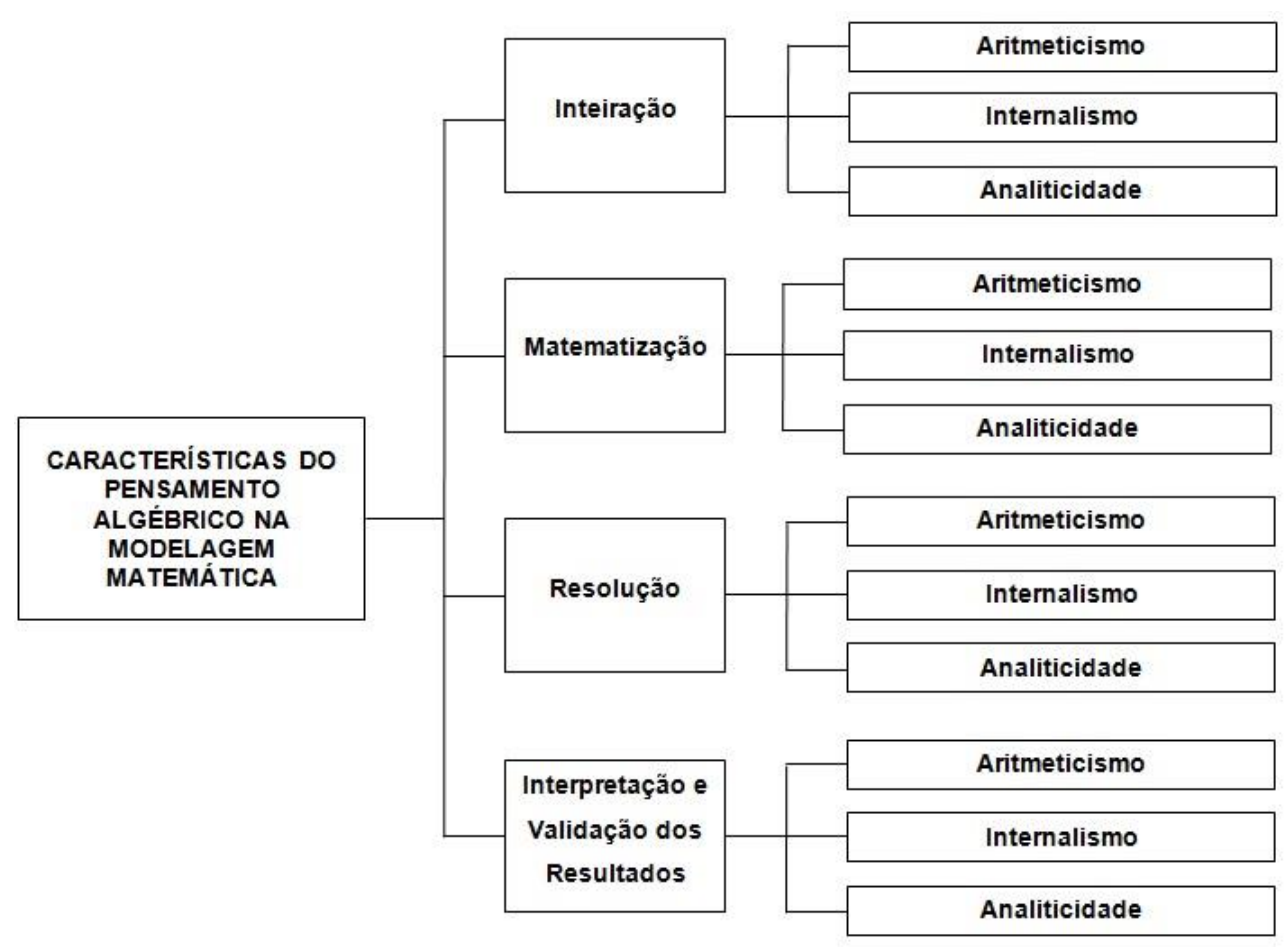

Fonte: Almeida, Silva e Vertuan (2012).

Neste texto, apresentamos a análise de cada unidade de análise, bem como as subunidades, as quais permitiram a construção de um metatexto que visa expressar "a compreensão do pesquisador sobre os significados e sentidos construídos" a partir dos textos originais (MORAES; GALIAZZI, 2011, p. 31).

Devido à limitação do artigo, será apresentado, em cada subunidade de análise, apenas o registro de um dos alunos que se enquadre na mesma. Os demais registros serão indicados somente pelos códigos.

\section{Unidade de análise efetiva: inteiração}

Esta unidade de análise evidencia a presença da inteiração, primeira fase da resolução de uma atividade por meio da modelagem matemática nas produções dos alunos. Nesta fase, os alunos procuraram entender a situação-problema, identificando as hipóteses e definindo as variáveis.

Essa unidade, assim como as demais, está subdividida em três subunidades previamente definidas referentes às características do pensamento algébrico evidenciadas neste momento da atividade de modelagem matemática. Das três subunidades de análise, duas se efetivaram: aritmeticismo (Quadro 1) e analiticidade (Quadro 2). 
Quadro 1: Subunidade Aritmeticismo

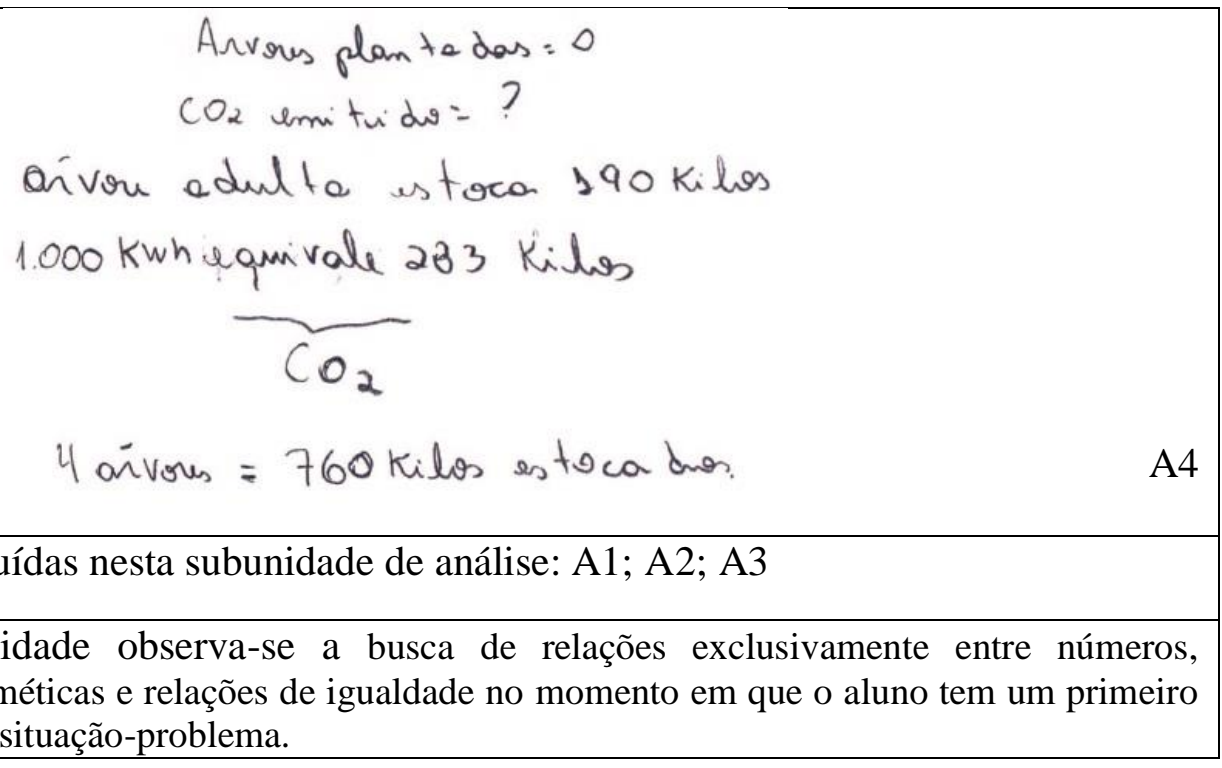

Fonte: Elaborado pelos autores.

Quadro 2: Subunidade Analiticidade

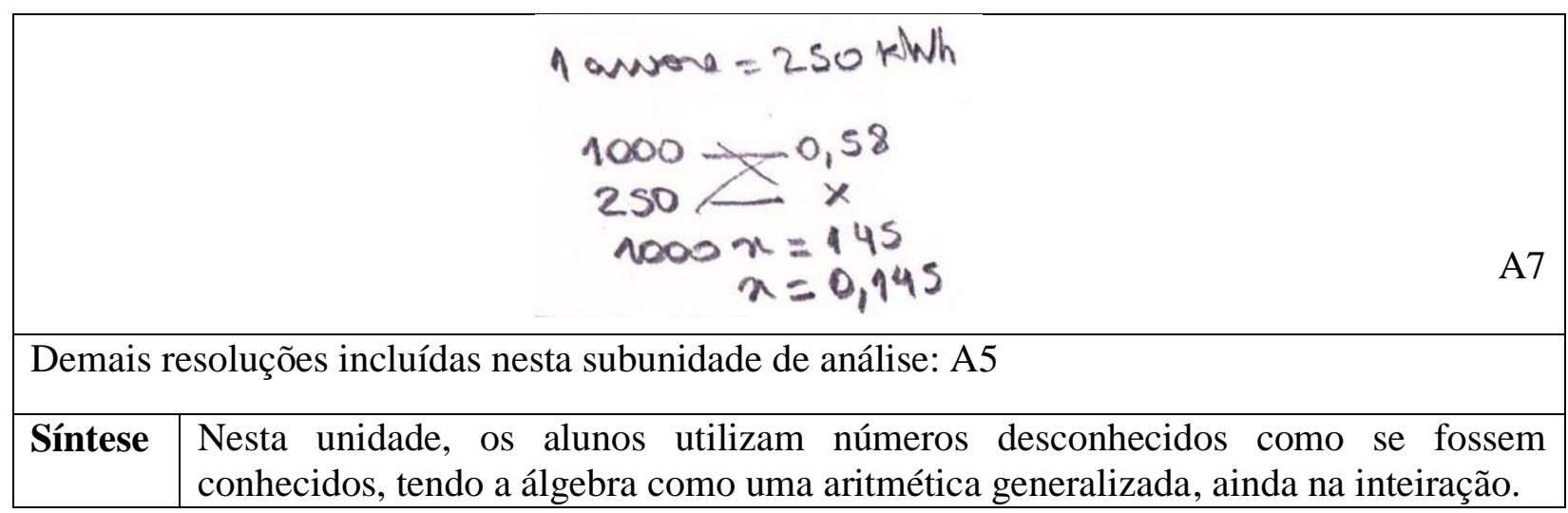

Fonte: Elaborado pelos autores.

\section{Unidade de análise efetiva: matematização}

Esta unidade de análise evidencia a matematização, a transição da linguagem natural para a linguagem matemática, denominada de matematização. Três características associadas ao pensamento algébrico dos alunos emergiram (Quadro 3, Quadro 4 e Quadro 5).

\section{Quadro 3: Subunidade Aritmeticismo}

$$
\begin{aligned}
& 100 \mathrm{omw} / \mathrm{h}=283 \mathrm{KgdeCO} \rightarrow 5 \text { arrones } \\
& 100 \mathrm{Kg}=0,1 \mathrm{~T} \mathrm{CO}_{2} \\
& \sum_{\mathrm{CO}_{2}=10,15 \quad \sum_{\text {invor: }} 64}
\end{aligned}
$$

Demais resoluções incluídas nesta subunidade de análise: A1; A2; A3; A4; A5; A7 
Síntese $\quad$ O aritmeticismo, ou seja, a produção de significados para números e operações algébricas pode ser visto por meio dos registros de todos os alunos, provavelmente devido ao contato desde os anos iniciais do Ensino Fundamental com a aritmética.

Fonte: Elaborado pelos autores.

Quadro 4: Subunidade Internalismo

$$
\begin{gathered}
m=\frac{y_{2}-y_{1}}{x_{2}-x_{1}} \\
m=\frac{0,87-0,58}{5-9} \quad m=0,29 \\
m=\frac{1,16-0,87}{7-5} \quad m=0,145 \\
m=\frac{2,32-0,58}{15-4} \quad m=0,158
\end{gathered}
$$

Demais resoluções incluídas nesta subunidade de análise: A5

Síntese $\quad$ Dos 7 alunos participantes da pesquisa, 2 deles (A5 e A6) apresentaram características de internalismo no momento da matematização. Os mesmos consideraram os números e as operações segundo suas propriedades, ou seja, há um estudo das estruturas matemáticas sem, necessariamente, considerar a situação inicialmente apresentada.

Fonte: Elaborado pelos autores.

Quadro 5: Subunidade Analiticidade

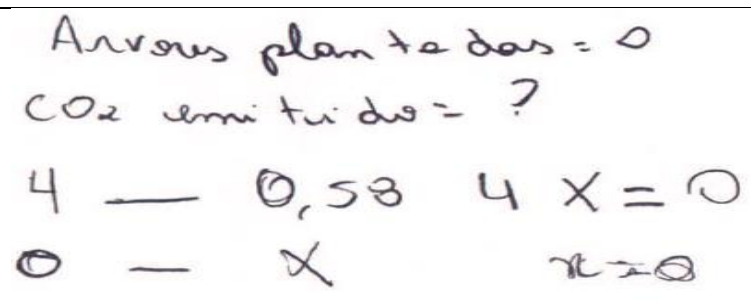

Demais resoluções incluídas nesta subunidade de análise: A5; A6

Síntese $\quad$ Nessa subunidade de análise foram incluídos os registros em que se observou a analiticidade no momento da matematização.

Fonte: Elaborado pelos autores.

\section{Unidade de análise efetiva: resolução}

Nesta fase da modelagem matemática, os alunos elaboram e apresentam um modelo matemático que represente e solucione a situação-problema proposta. Assim como na etapa anterior (matematização), todas as subunidades de análise previamente definidas se efetivaram (Quadro 6, Quadro 7, e Quadro 8). 
Quadro 6: Subunidade Aritmeticismo

$$
\begin{aligned}
& \frac{1500-1000}{5-4}=500=\frac{2000-1500}{7-5}=\frac{500}{2}=5 ., \\
& \frac{2500-0000}{9-7}=\frac{500}{2}=20
\end{aligned}
$$

Demais resoluções incluídas nesta subunidade de análise: A1; A2; A4; A5; A6; A7

\begin{tabular}{|l|l|}
\hline Síntese & $\begin{array}{l}\text { Todos os alunos chegaram a um modelo para a situação-problema inicialmente } \\
\text { proposta. Além disso, todos eles apresentaram, em seus registros, características do } \\
\text { aritmeticismo. }\end{array}$ \\
\hline
\end{tabular}

Fonte: Elaborado pelos autores.

Quadro 7: Subunidade Internalismo

$$
\begin{array}{lc}
y-y_{0}=m\left(x-x_{0}\right) & y-3,44 x-2,01=0 \\
y-4-3,44(x-0,58) & 0-3,44 x-2,04 \\
y-4=3,44 x-1,94 & x=\frac{2,04}{3,444}=0,58 \\
y-3,44 x=-0,01 &
\end{array}
$$

Demais resoluções incluídas nesta subunidade de análise: A2; A5; A6; A7

Síntese Nessa subunidade estão contempladas as resoluções que apresentaram, na etapa da resolução, a característica do pensamento algébrico denominada internalismo.

Fonte: Elaborado pelos autores.

Quadro 8: Subunidade Analiticidade

$$
\begin{array}{rl}
m=\frac{7-5}{1,86-0,87} m=6,89 & y=6,89 x-0,98 \\
y-11=6,89(x-1,74) \quad 0=6,89 x-0,98 \\
x=0,14
\end{array}
$$

Demais resoluções incluídas nesta subunidade de análise: A3; A5; A7

Síntese $\quad$ Nessa subunidade de análise foram incluídos os registro em a analiticidade foi evidenciada no momento da resolução.

Fonte: Elaborado pelos autores. 


\section{Unidade de análise efetiva: interpretação e validação dos resultados}

$\mathrm{Na}$ fase de interpretação e validação dos resultados, os alunos analisam se o resultado obtido com o modelo determinado relaciona-se corretamente com a situação estudada. Nesta unidade de análise, apenas duas, das três subunidades prévias, se efetivaram (Quadro 9 e Quadro 10).

Quadro 9: Subunidade Aritmeticismo

\begin{tabular}{|c|c|c|}
\hline & 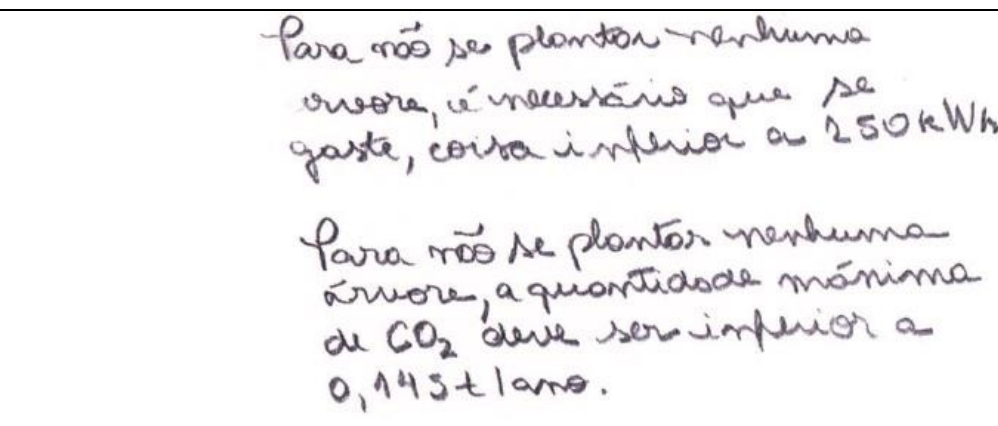 & A7 \\
\hline \multicolumn{3}{|c|}{ Demais resoluções incluídas nesta subunidade de análise: A2; A3 } \\
\hline Síntese & $\begin{array}{l}\text { Nessa subunidade estão contemplados os registros dos a } \\
\text { entre números, operações aritméticas e relações de igualdade no } \\
\text { da validação dos resultados obtidos. }\end{array}$ & ẫoes \\
\hline
\end{tabular}

Fonte: Elaborado pelos autores.

Quadro 10: Subunidade Analiticidade

\begin{tabular}{|l|}
\hline $\begin{array}{l}-6,32,4+y+24,7,0 \\
-25,28+24,7,-y\end{array}$ \\
$-0,58=-y$ \\
$y=0,58$
\end{tabular}

Fonte: Elaborado pelos autores.

\section{Metatexto: síntese da atividade dos alunos}

Para esta análise, uma categoria prévia foi estabelecida a fim de, por meio da análise textual discursiva, identificar características do pensamento algébrico no desenvolvimento de atividades de modelagem matemática. Uma categoria prévia características do pensamento algébrico na modelagem matemática foi analisada de modo a propiciar a elaboração teórica em torno da pergunta 
de pesquisa. Sua análise se deu por meio de quatro unidades de análise, a saber: inteiração; matematização; resolução; e interpretação e validação dos resultados, baseadas nas fases da modelagem matemáticas apresentadas por Almeida, Silva e Vertuan (2012).

Com vistas à identificação de características do pensamento algébrico em cada fase da atividade de modelagem matemática, cada unidade foi subdividida em três subunidades de análise, aritmeticismo, internalismo e analiticidade, sendo essas as características do pensamento algébrico, apontadas por Lins e Giménez (1997).

No início da atividade, na fase em que os alunos se inteiram da situação-problema, ou seja, buscam compreender o que é pedido, qual o fenômeno que está sendo descrito na situação, bem como compreender os dados da mesma, foi possível notar registros que indicam utilização de pensamentos relacionados ao aritmeticismo e à analiticidade. $\mathrm{O}$ uso da aritmética é feito desde os anos iniciais do Ensino Fundamental, o que torna compreensível tal característica aparecer logo no início da atividade de modelagem. É possível que o primeiro passo para entender a atividade por meio de uma linguagem matemática seja pensar nela por meio da utilização de números. Entendemos que o pensamento algébrico se dá também na relação que os mesmos estabelecem entre aritmeticismo, analiticidade e internalismo. A analiticidade pode ser vista quando os alunos leem a situação-problema já procurando estabelecer relações matemáticas nos dados que a compõem.

Na primeira fase da modelagem matemática, características associadas ao internalismo não se manifestaram, provavelmente devido ao fato de que o internalismo consiste em considerar números e operações apenas segundo suas propriedades sem, necessariamente, relacionar-se com a situação-problema, porém a inteiração é justamente o momento em que se busca conhecer especificidades e características da situação que se pretende estudar.

$\mathrm{Na}$ fase em que os alunos transitam da linguagem natural para a linguagem matemática, ou seja, na matematização, foi possível identificar todas as características do pensamento algébrico disponíveis na literatura: o aritmeticismo foi evidenciado na resolução dos 7 alunos, o internalismo em 2 resoluções e a analiticidade em 3 resoluções. A predominância, ainda neste momento, se restringe ao aritmeticismo.

É na fase de resolução que podemos observar a emergência das demais características. O aritmeticismo, aliado à analiticidade e ao internalismo é contemplado nos registros dos alunos. A produção de significados para a situação-problema e para a matemática que a soluciona é vista nos registros, em particular, de dois alunos A5 e A7, cujos registros manifestaram todas as características do pensamento algébrico descritas pela literatura. 
Em todas as fases da modelagem matemática é possível notar que os alunos fazem uso da matemática, porém, nas fases referentes à matematização e resolução, essas operações se tornam evidentes, uma vez que são nesses momentos que o modelo matemático é definido e a situaçãoproblema resolvida.

Além disso, foi possível observar que no momento da construção do modelo, etapa chamada de resolução, o internalismo mais se evidenciou. Possivelmente isso ocorreu pelo fato de os alunos lançarem mão de procedimentos matemáticos conhecidos por eles para a formulação do modelo. Dentre tais procedimentos estão resolução de sistemas lineares, definição de equação da reta, ajustes de funções, entre outros. Sendo assim, utilizar um procedimento matemático préestabelecido possibilitou aos alunos 'desvincularem-se' da situação-problema inicial e operar com os números e suas propriedades.

Com relação à última unidade de análise definida, interpretação e validação dos resultados, apenas 2 alunos a apresentaram na resolução. Destes, ambos evidenciaram as características aritmeticismo e analiticidade nesta etapa do desenvolvimento da atividade de modelagem matemática.

Vale destacar que se trata de uma turma do Ensino Médio e que os alunos não possuem contato constante com a modelagem matemática. Sendo assim, em nenhum momento foi exigido que eles apresentassem explicitamente, por exemplo, o registro do modelo obtido, a validação ou aplicação dos resultados. Mesmo assim, devido à estrutura da atividade de modelagem, essas fases emergem no desenvolvimento da mesma.

Ainda com relação às etapas da modelagem matemática, observa-se que as mesmas não ocorreram, especificamente, de maneira linear. Nos registros do aluno A6, por exemplo, é possível encontrar a validação de dois modelos matemáticos. Uma vez que o primeiro modelo encontrado pelo aluno não condizia com os dados apresentados pela situação-problema, o mesmo refez o processo de modelagem matemática, retornando à etapa da matematização, reescrevendo a situaçãoproblema em linguagem matemática, e determinou um segundo modelo matemático que foi validado e apresentado no quadro 10. Já que foi possível encontrar características da analiticidade nesta validação, este processo de retornar a etapas anteriores contempla a dinamicidade da atividade de modelagem matemática como proposto por Almeida, Silva e Vertuan (2012).

O desenvolvimento da atividade de modelagem matemática sugere que o pensamento algébrico de alunos, nesse tipo de atividade, está associado à produção de significados para a álgebra por meio do aritmeticismo, internalismo e analiticidade, seja pelo uso de números e operações algébricas, pela consideração exclusiva de conceitos matemáticos ou pela realização de 
operações. Da situação-inicial associada à plantação de árvores à situação-final, os alunos utilizam, operam, segundo essas características.

Assim, consideramos que atividades de modelagem matemática colocam os estudantes a investigar regularidades, sistematizar propriedades, resolver e discutir problemas algébricos, modelar situações e estabelecer padrões entre diferentes informações, indo ao encontro das atividades indicadas por Lins e Giménez (1997).

\section{Considerações finais}

Esse artigo nasceu do interesse em identificar características do pensamento algébrico evidenciadas no desenvolvimento de atividades de modelagem matemática. Para isso, buscou-se, por meio da análise dos dados coletados, investigar em quais fases da modelagem matemática, segundo Almeida, Silva e Vertuan (2012), as características do pensamento algébrico apresentadas por Lins e Giménez (1997) estão presentes.

Procuramos evidenciar o percurso metodológico dos pesquisadores, bem como o uso da metodologia de análise de dados, análise textual discursiva, que se mostrou positiva por permitir a criação de uma categoria a priori, com base no referencial teórico, de modo a contemplar o objetivo da pesquisa. A análise das unidades de análise e subunidades possibilitou compreender as características do pensamento algébrico que emergem no desenvolvimento de uma atividade de modelagem matemática por alunos.

A criação do metatexto permitiu avançar a análise da categoria, partindo para uma compreensão sob a combinação dos elementos construídos na etapa anterior.

Com a análise foi possível perceber que, no desenvolvimento de uma atividade de modelagem matemática, características do pensamento algébrico, como aritmeticismo, internalismo e analiticidade, podem emergir nas diferentes etapas desta alternativa pedagógica, mesmo que por diferentes alunos e em diferentes momentos. Além disso, observou-se que a característica aritmeticismo foi evidenciada mais vezes, seguida pela analiticidade e pelo internalismo, respectivamente. O internalismo foi evidenciado nas etapas de matematização e resolução.

Assim, inferimos que atividades de modelagem matemática permitem que os alunos tenham a percepção de regularidades, aspectos invariantes e que variam, busquem expressar ou explicitar a estrutura de uma situação-problema e a presença do processo de generalização, sendo estes os caracterizadores do pensamento matemático aqui estudado. 


\section{Referências}

ALMEIDA, L. M. W.; BRITO , D. S.. Atividades de Modelagem Matemática: que sentido os alunos podem the atribuir?.Ciência e Educação (UNESP), 11, 1-16, 2005.

; SILVA, K. P.; VERTUAN, R. E. Modelagem Matemática na Educação Básica. São

Paulo: Contexto, 2012.

ARAÚJO, J. L. Uma Abordagem Sócio-Crítica da Modelagem Matemática: a perspectiva da educação matemática crítica. Alexandria: Revista de Educação em Ciência e Tecnologia, v. 2, n. 2, p. 55-68, 2009.

BARBOSA, E.; BORRALHO, A. Exploring Patters and Algebraic Thinking.Conference of the International Group of the Psychology of Mathematics Education, 1, 344, 2009.

BARBOSA, J. C. Modelagem na Educação Matemática: contribuições para o debate teórico. In: Reunião anual da Anped, 24, 2001, Caxambu. Anais... Rio de Janeiro: ANPED, 2001. CD-ROM.

BEAN, D. Modelagem na perspectiva do pensamento. In: III Conferência Nacional sobre Modelagem e Educação Matemática, 2003, Piracicaba - SP. Anais da III Conferência Nacional sobre Modelagem e Educação Matemática, 2003.

BIEMBENGUT, M. S. 30 Anos de Modelagem Matemática na Educação Brasileira: das propostas primeiras às propostas atuais. Alexandria - Revista de Educação em Ciência e Tecnologia. v. 2, n. 2, p. 7-32, jul. 2009.

CARRAHER, D. W.; SCHLIEMANN, A.; BRIZUELLA, B. Early Arithimetic: Treating operations as functions. Plenary presentation at PME - NA XXII, Tucson, AZ, 2006.

CYRINO, M. C. C. T; OLIVEIRA, H. M. Pensamento Algébrico ao longo do Ensino Básico em Portugal. Bolema, Rio Claro (SP), v. 24, n 38, pp. 97-126, abril 2011.

FERRI, R. B. Theoretical and empirical differentiations of phases in the modeling process. ZDM The International Journal on Mathematics Education, v. 38, n. 2, p. 86-95, 2006.

FIORENTINI, D.; FERNANDES, F. L. P.; CRISTOVÃO, E. M. Um estudo das potencialidades pedagógicas das investigações matemáticas no desenvolvimento do pensamento algébrico.

Seminário Luso-brasileiro de investigações matemáticas no currículo e na formação do professor, 2005.

; MIORIM, M. A.; MIGUEL, A. Contribuição para um repensar... a educação algébrica elementar. Revista Pro-Prosições, 79-91, 1993.

GALBRAITH. Models of Modelling: Genres, Purposes or Perspectives. Journal of Mathematical Modelling and Application, vol. 1, n. 5, 3-16, 2012.

KAISER, G.; B. SRIRAMAN. A global survey of international perspectives on modelling in mathematics education. ZDM, 38, 3, 302-310, 2006.

KAPUT, J. J. Teaching and learning a new algebra. In: FENNEMA, E.; ROMBERG, T. (Eds.), Mathematics classrooms that promote understanding. Mahwah, NJ: Erlbaum, p. 133-155, 1999.

KIERAN, C. Developing algebraic reasoning: The role of sequenced tasks and teacher questions from the primary to the early secondary school levels. Quadrante, XVI, 1, 5-26, 2007.

KLÜBER, T. E. Aspectos relativos à noção de prática(s) de modelagem matemática na educação matemática. Revemat, v. 08, n. 1, p. 92- 103, 2013.

LINS, R. C. Matemática, monstros, significados e educação matemática. Educação matemática: pesquisa em movimento. São Paulo: Cortez, 2004. 
; J. GIMÉNEZ. Perspectivas em aritmética e álgebra para o século XXI. Campinas:

Papirus Editora, 1997.

LUCCAS, S. O ensino introdutório de matemática em cursos de administração: construção de uma proposta pedagógica. Tese de Doutorado em Ensino de Ciências e Educação Matemática, Universidade Estadual de Londrina (Brasil), Centro de Ciências Exatas, 2011.

MORAES, R.; GALIAZZI, M. C. Análise textual discursiva. Ijuí: Unijuí, 2007.

PARANÁ. Secretaria de Estado da Educação. Diretrizes Curriculares da Educação Básica do Paraná. Curitiba: MEC, 2008.

PONTE, J. P. O ensino da matemática em Portugal: Uma prioridade educativa? O ensino da Matemática: Situação e Perspectiva (21-56). Lisboa: Conselho Nacional de Educação, 2003.

SKOVSMOSE, O. Desafios da reflexão em educação matemática crítica. Campinas: Papirus Editora, 2008. 